\title{
Assessment of nonsteroidal anti-inflammatory drug combinations by the polyurethane sponge implantation model in the rat
}

\author{
R. GARRETT, B. MANTHEY, B. VERNON-ROBERTS, AND P. M. BROOKS * \\ From the Adelaide Rheumatology Research Group, Department of Pathology, University of Adelaide, and the \\ ${ }^{*}$ Department of Medicine, Flinders University, South Australia
}

SUMmaRY The anti-inflammatory effect of single doses and combinations of aspirin and commonly used nonsteroidal anti-inflammatory drugs was investigated by the polyurethane sponge implantation model of acute inflammation. Dose response curves were performed to delineate a suppressive (high) and nonsuppressive (low) dose of each drug prior to studying these doses in combination with aspirin. The results suggest that a combination of aspirin in either high or low dose does not increase the anti-inflammatory effect of other nonsteroidal anti-inflammatory drugs in this model of inflammation.

Nonsteroidal anti-inflammatory drugs (NSAID) still form the basis of treatment for inflammatory rheumatic diseases, and a significant number of patients with rheumatoid arthritis or osteoarthritis still receive more than one of these agents at the same time. ${ }^{1}$ Pharmacokinetic interactions between NSAIDs have been described frequently in man. ${ }^{2-5}$ Few studies have been done on the clinical aspects of such interactions and those that have reveal conflicting results. Recently combinations of ibuprofen and aspirin $^{6}$ and indomethacin and low-dose sodium salicylate $^{7}$ have demonstrated greater effectiveness of the combination than either drug alone. On the other hand combinations of indomethacin and aspirin, ${ }^{8}$ flurbiprofen and aspirin, ${ }^{4}$ and flurbiprofen and indomethacin ${ }^{9}$ have failed to demonstrate any additional anti-inflammatory effect of these combinations over the single agents.

Van Arman et al. ${ }^{10}$ have shown antagonism between aspirin and a variety of NSAIDs on adjuvant induced arthritis in the rat, and the antagonism of NSAID combinations has also been shown on carrageenen-induced oedema in the rat by Mielins $e t$ $a l .{ }^{11}$ and Swingle et al. ${ }^{12}$ These workers used singledose combinations of NSAID in their experiments. We decided therefore to study the effect of varying doses of commonly used NSAIDs in combination with varying doses of salicylate in the polyurethane

Accepted for publication 22 June 1982.

Correspondence to Dr P. M. Brooks, Department of Medicine, Flinders Medical Centre, Bedford Park, South Australia 5042. sponge implantation model. ${ }^{13}$ The advantage of this model is that it allows both the fluid and cellular phases of the inflammatory reaction to be studied separately. Results reported here relate to the effect of NSAID combinations on the fluid phase of inflammation only.

\section{Materials and methods}

Polyurethane foam, $7 \mathrm{~mm}$ in thickness, was cut to $15 \times 15 \mathrm{~mm}$ blocks with an average weight of $40 \mathrm{mg}$, the weight of the sponges varying by only $1 \mathrm{mg}$ for each experiment. These sponges were then impregnated with heat-killed human strain Mycobacterium tuberculosis by immersing and compressing them in an aqueous suspension of Myco. tuberculosis at a concentration of $0.5 \mathrm{mg} / \mathrm{ml}$ followed by air drying overnight at $37^{\circ} \mathrm{C}$ to constant weight. Before implantation the cubes were sterilised at $121^{\circ} \mathrm{C}$ for $15 \mathrm{~min}$. One impregnated sponge was then implanted into each flank of $150-250 \mathrm{~g}$ DA inbred rats through a midline dorsal incision under ether anaesthesia by a sterile technique. Forty animals with implanted sponges were divided into 5 groups of 8 animals, each group receiving different drug combinations. During the following 4 days the animals received combined doses of aspirin and other NSAID orally suspended in carboxymethyl cellulose as shown in Table 1 . The doses chosen were a high and low dose selected from dose response curves. The low dose was one that did not significantly reduce inflammation over control, 
Table 1 Drug doses used ( $\mathrm{mg} / \mathrm{kg} /$ day)

\begin{tabular}{lll}
\hline & $\begin{array}{l}\text { Low dose } \\
\text { (nonsuppressive) }\end{array}$ & $\begin{array}{l}\text { High dose } \\
\text { (suppressive) }\end{array}$ \\
\hline Aspirin & 20 & 200 \\
Indomethacin & $0 \cdot 05$ & 4 \\
Naproxen & $0 \cdot 25$ & 20 \\
Ibuprofen & 5 & 60 \\
Fenoprofen & 1 & 15 \\
Diclofenac & $0 \cdot 2$ & 3 \\
Flurbiprofen & $0 \cdot 3$ & 3 \\
\hline
\end{tabular}

while the high dose did produce significant reduction in weight gain of the sponge. The dose response curves determined prior to each experiment are shown in Fig. 1. Untreated controls in each group received carboxymethyl cellulose alone.

The animals were killed 5 days after sponge implantation and the sponges were removed. One sponge from each of 2 animals in each treatment group was used for histology. The remaining 14 sponges in each group were used to study sponge weight gain, and during removal care was taken not to squeeze any of the contained fluid. These sponges were then dried overnight to constant weight at $37^{\circ} \mathrm{C}$. The difference between the initial and final dry weight of each sponge was recorded as the dry weight gain. This reflects the fluid phase of the inflammatory exudate ${ }^{13}$ The reduction in dry weight gain of each treated group in relation to control group of each experiment was calculated and recorded to assess the anti-inflammatory effect of the drug combination.

Student's $t$ test for unpaired values was used to assess differences between treated and control groups for each set of experiments.

\section{Results}

The results of the dry weight gain of the sponge seen

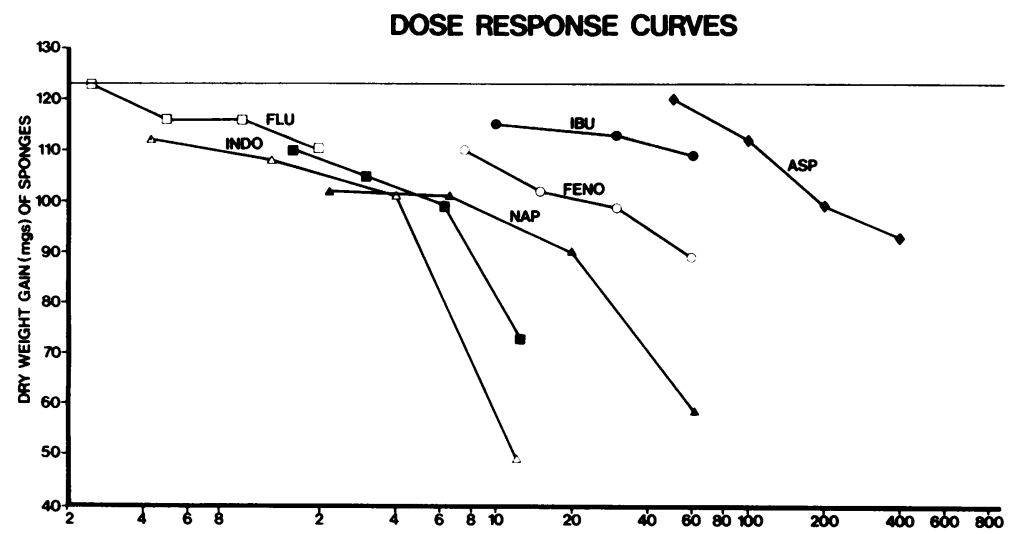

Fig. 1 Dose response curves for aspirin and NSAIDs.

Table 2 Dry weight gain of impregnated cube seen with each combination of nonsteroidal anti-inflammatory drug (mg) $($ mean $\pm S E)$

\begin{tabular}{|c|c|c|c|c|c|c|c|c|c|}
\hline Group & 1 Control & & $\begin{array}{l}2 \\
\text { NS aspirin } \\
\text { NS drug }\end{array}$ & & $\begin{array}{l}3 \\
\text { NS aspirin } \\
S \text { drug }\end{array}$ & & $\begin{array}{l}4 \\
S \text { aspirin } \\
\text { NS drug }\end{array}$ & & $\begin{array}{l}5 \\
S \text { aspirin } \\
S \text { drug }\end{array}$ \\
\hline Naproxen & $121 \pm 0 \cdot 5$ & 1 & $121 \pm 2 \cdot 5$ & $p<0.0005$ & $110 \pm 1 \cdot 3$ & $p<0.0005$ & $110 \pm 2 \cdot 6$ & $p<0.0005$ & $105 \pm 1$ \\
\hline Indomethacin & & NS & & $\mathrm{p}<0.0005$ & & $p<0.0005$ & $109 \pm 0.6$ & $p<0.05$ & $121 \pm 4 \cdot 9$ \\
\hline Fenoprofen & $123 \pm 2 \cdot 1$ & NS & $122 \pm 2 \cdot 9$ & $p<0.025$ & $116 \pm 1 \cdot 2$ & $p<0.0005$ & $102 \pm 4 \cdot 6$ & $p<0.0005$ & $\bullet 101 \pm 0.1$ \\
\hline Ibuprofen & & NS & $117 \pm 2 \cdot 8$ & $p<0.0005$ & $114 \pm 0 \cdot 8$ & $p<0.0005$ & $97 \pm 5 \cdot 2$ & $p<0.0005$ & $100 \pm 1 \cdot 3$ \\
\hline Diclofenac & $110 \pm 5 \cdot 1$ & $\mathrm{p}<0.025$ & $105 \pm 1 \cdot 0$ & $p<0.0025$ & $95 \pm 3 \cdot 3$ & $p<0.005$ & $103 \pm 6 \cdot 2$ & $p<0.005$ & $98 \pm 3 \cdot 3$ \\
\hline Flurbiprofen & $119 \pm 1 \cdot 5$ & $\mathrm{p}<0.05$ & $113 \pm 4 \cdot 0$ & $p<0.0005$ & $104 \pm 3 \cdot 5$ & $\mathrm{p}<0.0005$ & $108 \pm 1 \cdot 6$ & $p<0.0005$ & $102 \pm 3 \cdot 1$ \\
\hline
\end{tabular}

Significance by unpaired Student's $t$ test in comparison with control. 
with combination of aspirin with the 6 NSAIDs is summarised in Table 2. Figs. 2, 3, and 4 summarise the results graphically for the high-aspirin, lowNSAID combination (Fig. 2), the low-aspirin, highNSAID combination (Fig. 3), and the high-aspirin, high-NSAID combination (Fig. 4). It can be seen that, although significant reduction in dry weight gain was produced with combined drug therapy, the actual decrease in the values obtained in nearly all cases was significantly less than that produced when the drugs were given alone. The only exception to this was when high-dose aspirin and high-dose ibuprofen were combined.

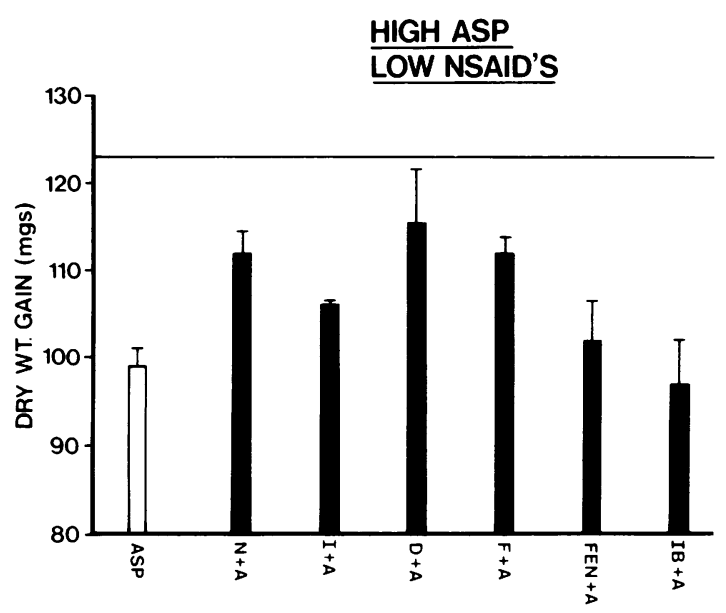

Fig. 2 Dry weight gain seen with high-aspirin and low-NSAID combination.

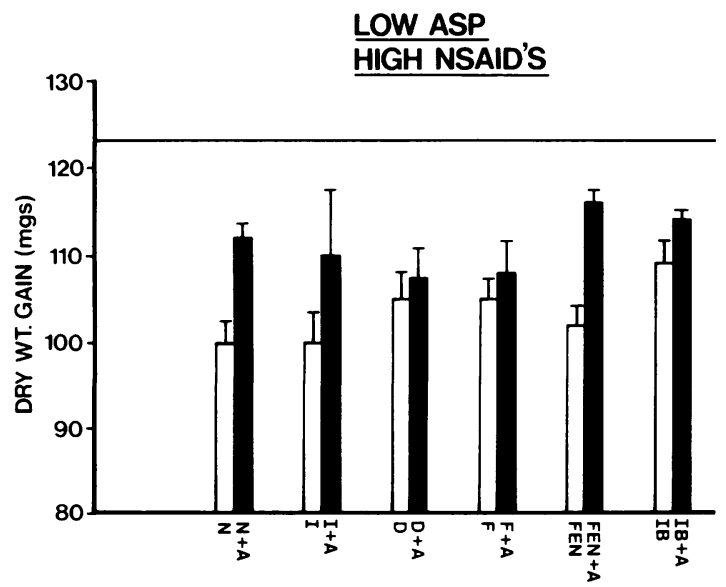

Fig. 3 Dry weight gain seen with low-aspirin and high-NSAID combination.

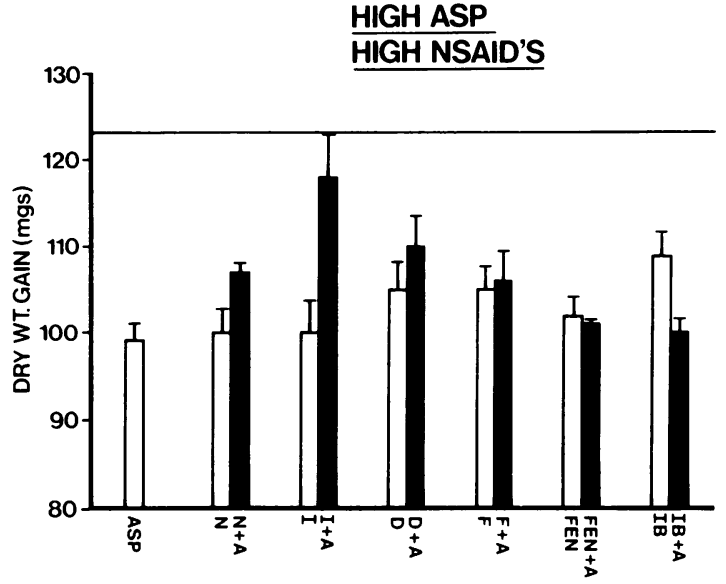

Fig. 4 Dry weight gain seen with high-aspirin and high-NSAID combination.

Low-dose aspirin combined with a low-dose NSAID did not significantly suppress weight gain relative to the control value except when combined with diclofenac or flurbiprofen. Low doses of aspirin antagonised the anti-inflammatory effects of high doses of NSAIDs (Fig. 3), and low doses of NSAIDs interfered with the anti-inflammatory effect of highdose aspirin (except for ibuprofen) as seen in Fig. 2. In Fig. 4 it can be clearly seen that high doses of NSAIDs antagonised the effects of high-dose aspirin in the case of naproxen and indomethacin or did not convey any additional anti-inflammatory effect in the case of diclofenac, fenoprofen, and flurbiprofen. The combination of high-dose ibuprofen and high-dose aspirin provided a significantly greater antiinflammatory effect than ibuprofen alone but not a significantly greater effect than aspirin alone.

\section{Discussion}

The impregnated polyurethane sponge model has been shown to be useful in measuring both the fluid and cellular phases of the acute inflammatory response. ${ }^{13}$ Though some variation does occur in the inflammatory reaction produced by this sponge due to minor variations in sponge preparation and between groups of rats (Table 2), sponges from the same batch were used for each set of interaction experiments. The present study has shown that all the NSAIDs investigated suppress the fluid phase of the acute inflammatory response and that most of the NSAIDs studied and aspirin interfere with the antiinflammatory effects of each other when taken alone. This is seen particularly when suppressive doses of aspirin are combined with suppressive doses of other 
NSAIDs. Thus the combination of high doses of indomethacin and aspirin do not reduce the dry weight gain to the same extent as low doses of aspirin plus high doses of indomethacin, or a high-dose of aspirin plus a low-dose of indomethacin. This antagonism of anti-inflammatory effect of NSAIDs by aspirin occurs with all drugs except ibuprofen. These data would seem to support the added antiinflammatory effect of aspirin and ibuprofen observed clinically. ${ }^{6}$ There seems to be no doubt that combinations of aspirin with NSAIDs do interfere with each other from the point of view of suppression of the fluid phase of inflammation but that these interactions occur on an individual basis. If a similar antagonism occurs in man, then with these rather unpredictable interactions it would seem logical to restrict the prescribing of NSAID combinations to situations where single drug therapy was proved ineffective in maximally tolerated doses and where a single acceptable alternative is not available.

We thank Merck Sharp and Dohme (Australia) Pty Ltd, Boots Australia, Syntex Australia, and Lilly Industries (Australia) for providing pure drugs. B. Manthey was supported by a grant from the South Australian Arthritis and Rheumatism Association.

\section{References}

1 Brooks P M, Bell M A, Mason D I R, Buchanan W W. Interactions of antirheumatic drugs. Agents Actions 1977; suppl 1: 85-95.
2 Rubin A, Rodda B E, Warrick P, Gruber C M, Ridolfo A S. Interactions of aspirin with non-steroidal anti-inflammatory drugs in man. Arthritis Rheum 1973; 16: 635-45.

3 Segre E J, Chaplin M, Forchielli E, Runkel R, Sevelius H. Naproxen-aspirin interactions in man. Clin Pharmacol Ther 1974; 15: 374-9.

4 Brooks P M, Khong T K. Flurbiprofen/aspirin interactions: a double blind crossover study. Curr Med Res Opin 1977; 5: 53-7.

5 Williams R L, Upton R A, Buskin J N, Jones R M. Ketoprofenaspirin interactions. Clin Pharmacol Ther 1981; 30: 226-31.

6 Grennan D M, Ferry D G, Ashworth M E, Kenny R E, Mackinnon $M$. The aspirin-ibuprofen interaction in rheumatoid arthritis. Br J Clin Pharmacol 1979; 8: 497-503.

7 Ezer E, Palosi E, Hajos G Y, Rosdy B, Szporny L. Comparative pharmacology of a 1:10 combination of indomethacin-sodium salicylate. Agents Actions 1979; 9: 119-23.

8 Brooks P M, Walker J J, Bell M A, Buchanan W W, Rhymer A R. Indomethacin-aspirin interaction: a clinical appraisal. $\mathrm{Br} \mathrm{Med}$ $J$ iii: 69-71.

9 Rudge S R, Lloyd-Jones J K, Hind I D. Interaction between flurbiprofen and indomethacin in rheumatoid arthritis. Br J Clin Pharmacol 1982; 13: 448-51.

10 Van Arman C G, Nuss G W, Risely E A. Interactions of aspirin, indomethacin and other drugs in adjuvant-arthritis in the rat. $J$ Pharmacol Exp Ther 1973; 187: 400-14.

11 Mielens Z E, Drobeck H P, Rozitis J K, Sansone J J Jr. Interactions of aspirin with non-steroidal anti-inflammatory drugs in rats. J Pharm Pharmacol 1968; 20: 567-9.

12 Swingle K F, Grant T J, Jaques L W, Kwam D C. Interactions of anti-inflammatory drugs on carageenen-induced foot oedema of the rat.J Pharmacol Exp Ther 1970; 172: 423-5.

13 Clarke A K, Vernon-Roberts B, Currey H L F. Assessment of anti-inflammatory drugs in the rat using subcutaneous implants of polyurethane foam impregnated with dead tubercle bacilli. Ann Rheum Dis 1975; 34: 326-31. 